\title{
Evidence for atomic processes in molecular valence double ionization
}

\author{
U. Becker, O. Hemmers, B. Langer, A. Menzel, and R. Wehlitz \\ Fritz-Haber-Institut der Max-Planck-Gesellschaft, W-1000 Berlin 33, Germany \\ W. B. Peatman \\ Berliner Elektronenspeicherring-Gesellschaft für Synchrotronstrahlung mit beschränkter Haftung, \\ $W$-1000 Berlin 33, Germany
}

(Received 21 June 1991)

\begin{abstract}
Complete molecular valence-electron spectra were measured for CO. Unexpectedly, discrete lines at low kinetic energies were found, superimposed on a continuous energy spectrum representing direct double-ionization processes. The appearance of these lines is discussed in the context of the formation of the $\mathrm{C}^{+}+\mathrm{O}^{+}$ion pair near its associated threshold at $38.4 \mathrm{eV}$. It is ascribed to valence-excited repulsive $\left(\mathrm{CO}^{+}\right)^{*}$ states, which dissociate to a large part rapidly into atomic fragments before electronic relaxation takes place. From our spectra, partial cross sections for the different processes leading to dissociative valence double ionization are derived.
\end{abstract}

PACS number(s): $33.60 . \mathrm{Cv}, 33.80 . \mathrm{Eh}, 33.80 . \mathrm{Gj}$

Photoionization of the inner-valence shells of most molecules is characterized by a very complex photoelectron spectrum due to the breakdown of the one-electron molecular-orbital model. For example, in $\mathrm{CO}$, all the ionic states in the inner-valence shell region have mixed configurations of the $3 \sigma$ single-hole state and the outervalence two-hole one-electron states [1]. In the case of double ionization, these "inner-valence" states are energetically degenerate with the lowest double-ionization threshold, which is in most cases a predissociated state of the molecular dication [2]. In this energy region ionization and excitation is, in contrast to processes involving core electrons, assumed to be not very site specific because of the heterogeneous localization of the involved electrons. This situation gives little rise to expect any site-specific effects; furthermore, it prevents a direct determination of the single-photon double-photoionization cross section of molecules from photoionization data [3,4]; more sophisticated methods such as coincidence experiments are required. Such a study, recently performed by Lablanquie et al. [5], revealed many details of the double ionization of $\mathrm{CO}$, including pathways to multiply charged molecular ions via certain electronic excitations indicating a high fractional intensity of these processes of approximately $25 \%$ at a photon energy of $80 \mathrm{eV}$. However, certain key points of our understanding of molecular valence photoionization and dissociation and their mutual relationship are still widely unknown, e.g., the character of the proposed two-step ionization mechanism for the formation of $\mathrm{C}^{+}+\mathrm{O}^{+}$ion pairs at threshold.

The interpretation of molecular double-ionization results requires the understanding of various effects, which are often obscured because of the many pathways by which most charged states and ionic fragments can be produced. For example, molecular double ionization is in many cases associated with the production of a pair of positively charged fragments, a process known as dissociative double ionization. Regarding nonresonant processes in $\mathrm{CO}$ this may occur either via direct double ionization,
$\mathrm{CO}+h v \rightarrow \mathrm{CO}^{2+}+2 e^{-} \rightarrow \mathrm{C}^{+}+\mathrm{O}^{+}+2 e^{-}$, or alternatively by indirect routes, such as (a) indirect double ionization followed by dissociation,

$$
\begin{aligned}
\mathrm{CO}+h v & \rightarrow\left(\mathrm{CO}^{+}\right)^{*}+e^{-} \rightarrow \mathrm{CO}^{2+}+2 e^{-} \\
& \rightarrow \mathrm{C}^{+}+\mathrm{O}^{+}+2 e^{-},
\end{aligned}
$$

and (b) direct dissociation of the molecular ion with subsequent relaxation in the excited atom,

$$
\begin{aligned}
\mathrm{CO}+h v & \rightarrow\left(\mathrm{CO}^{+}\right)^{*}+e^{-} \\
& \rightarrow \mathrm{C}^{+}+\mathrm{O}^{*}+e^{-} \rightarrow \mathrm{C}^{+}+\mathrm{O}^{+}+2 e^{-} .
\end{aligned}
$$

The two indirect processes differ basically from each other by the interatomic distance where the electronic relaxation occurs, (a) still in the molecule or (b) already in the fragments. It is well known from the decay of molecular core-electron excitations that fast dissociation may lead to electronic relaxation in the neutral atomic fragments, prominent examples of this behavior being $\mathrm{HBr}$ and $\mathrm{HI}[6,7]$. Process (b) represents the analog situation in the case of valence-electron double ionization. Depending on the time scale of the electronic relaxation there is a continuous transition between both types of relaxation processes giving rise to competition between them. Besides the clear evidence for atomic deexcitation of coreexcited molecules there was little known about similar behavior in the valence shells. On the contrary, most studies considering dissociative valence double ionization via two-step processes expect electronic relaxation to be much faster than the accompanying dissociation $[8,9]$. Furthermore, besides the question of atomic versus molecular relaxation, nearly nothing is known in molecules about the absolute ratios between simultaneous double ionization via shake-off and indirect pathways such as two-step processes.

In this Rapid Communication we report on a series of complete molecular valence-electron spectra of $\mathrm{CO}$ from near-zero volts up to the inner-valence photoline, taken 
using synchrotron-radiation excitation. Analogous to atomic photoionization studies [10-12], but in contrast to expectations regarding valence photoionization of molecules, we observe a discrete line spectrum in the lowenergy part of these spectra. These lines appear, also analogous to the atomic case, at the same kinetic energy for all photon energies above threshold suggesting an interpretation as Auger transitions. The main purpose of the experiment was to study the impact of molecular effects, such as dissociation, on the possible relaxation of two-hole-one-electron satellite states. In particular, the relative strength of three different processes, direct double ionization, indirect double ionization via molecular Auger decay, and dissociative double ionization via atomic relaxation in the fragments, should be determined.

We have recorded molecular valence-electron spectra of $\mathrm{CO}$ in the photon-energy range from $50-120 \mathrm{eV}$ with emphasis on electrons emitted with low kinetic energies. In order to assign the primary photoionization process in the inner-valence region, a high-resolution spectrum of the energy region of the so-called $3 \sigma$ peak in $\mathrm{CO}$ was taken unveiling a rich structure of satellite lines. These lines were tentatively designated according to calculations by Schirmer and Walter [13] and seem to correspond to the highly excited $\left(\mathrm{CO}^{+}\right)^{*}$ states predicted by Lablanquie et al. [5] on the basis of their ion-ion coincidence experiments. The character of these satellite states and their relation to the electrons observed at low kinetic energies is discussed with respect to their energy positions and widths considering the different dynamic aspects of molecular relaxation as described above.

The experiment was in the main part conducted on the 5.6-m toroidal grating monochromator (TGM) at the Hamburger Synchrotronstrahlungslabor HASYLAB utilizing the single-bunch timing mode of the electron storage ring DORIS II. This mode has a time window of 960 nsec, which is particularly suited for recording complete electron spectra with our time-of-flight electron spectrometer. Details of our spectrometer system and the beam line have been described elsewhere $[14,15]$. The experimental conditions were similar to those of our rare-gas experiments [10]. One highly resolved spectrum of $\mathrm{CO}$ was taken at the undulator-wiggler beam line of the Berliner Speicherringgesellschaft für Synchrotronstrahlung (BESSY) [16].

All former molecular valence-photoelectron spectra were limited with respect to two points: (i) the coverage of the energy range of the electrons emitted and (ii) the resolution of the inner-valence peak structure. Therefore, there was no evidence on the electron spectrometry side for sequential processes and discrete satellite structure above the dissociative double-ionization threshold, although there was indirect evidence for the existence of such states [5].

Figure 1 shows a series of complete electron spectra for CO. These spectra show, for valence photoionization, an unexpectedly strong appearance of electron lines staying at fixed kinetic energies independent of the excitation energy. These narrow lines are concentrated at low kinetic energies, raising the question of their origin. In order to explain the formation of $\mathrm{C}^{+}+\mathrm{O}^{+}$ion pairs at a threshold

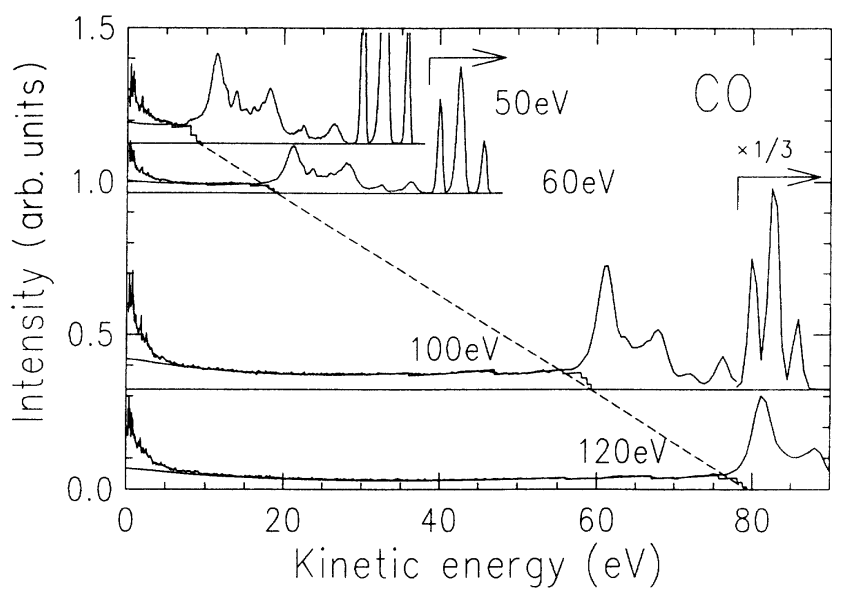

FIG. 1. Series of complete molecular valence-electron spectra of $\mathrm{CO}$ taken at various photon energies. The solid curves represent the theoretical shake-off distributions of Chang and Poe (Ref. [26]) adjusted to the experimental spectra as described in the text.

energy of $38.4 \mathrm{eV}$, Lablanquie et al. [5] proposed the existence of highly excited $\left(\mathrm{CO}^{+}\right)^{*}$ states, which decay into the double-ionization continuum. If this mechanism describes the dissociative double ionization near threshold correctly, there should exist a variety of $\left(\mathrm{CO}^{+}\right)^{*}$ states above this threshold in the binding-energy spectrum of the $3 \sigma$ peak. Because there was little evidence for such states in inner-valence photoelectron spectra to date [17], we recorded this energy region of the valence-photoelectron spectrum of $\mathrm{CO}$ at high resolution using the undulatorwiggler beam line of BESSY [16]. The result of this undulator measurement is displayed in Fig. 2, revealing many discrete lines exhibited on the broad structure of the so-called $3 \sigma$ peak. Closer inspection of these lines shows some indications for a possible correspondence between the structure seen on the $3 \sigma$ peak and the fixed low-energy line structure. Such a correspondence would be formally analogous to the atomic case [10], although there is no ob-

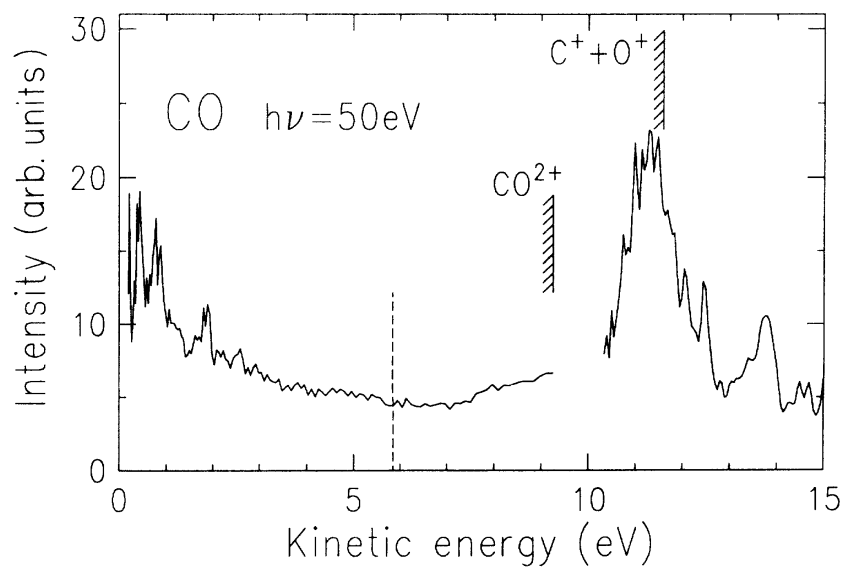

FIG. 2. High-resolution $3 \sigma$ undulator spectrum taken at a photon energy of $50 \mathrm{eV}$ together with the low-energy part of the corresponding complete valence spectrum of Fig. 1 (solid line below the $\mathrm{CO}^{2+}$ threshold). 
vious reason to expect this correspondence generally in case of a molecule. Because nothing is known about the nature of these $\mathrm{CO}^{+}$states, we may assume that these low-kinetic-energy electrons result from process (a) [5]. However, would molecular Auger decay to the dissociative $\mathrm{CO}^{2+}{ }^{3} \Sigma^{-}$state give rise to a discrete line spectrum as observed in our spectra? The answer is probably not, because decay to a repulsive molecular state would lead to a quasicontinuum of unresolved and broadened electron lines [18]. Therefore we propose mechanism (b)-the direct dissociation following single ionization with subsequent relaxation in one fragment atom, $\mathrm{C}^{*}$ or $\mathrm{O}^{*}$ - as the origin of the discrete part of the low-energy electrons. This interpretation is consistent with three characteristic features of our electron spectra: (i) a discrete electron line spectrum with line widths of less than $0.1 \mathrm{eV}$, (ii) the lines appear at fixed kinetic energy independent of the excitation energy and are related to excited states in the neutral atoms, (iii) their appearance depends on the existence of satellite states in the energy region of the $3 \sigma$ peak above the experimentally observed dissociative double-ionization threshold. The fact that this process appears also at near threshold kinetic energy of the electron shows that the remaining energy is given completely as kinetic energy to the nuclei promoting fast dissociation of the molecule as suggested for the interpretation of our spectra.

In light of the above-mentioned possible correspondence between first and second step transition lines the interpretation of the observed discrete lines as being of atomic origin raises the question how such an eventual relation between those atomic lines and their primary molecular two-hole-one-electron satellite lines could occur. Two aspects are important to consider in trying to understand this situation. (i) The variety of atomic lines observed suggests that each molecular satellite state does populate a different excited atomic state or at least that several such states are populated via the dissociation process crossing the lowest $\mathrm{CO}^{2+}$ state. (ii) The fixed kinetic energy of the dissociating fragments [5] may indicate that the energy positions of the molecular excitations keep related to each other during dissociation evolving in a corresponding set of atomic excitations as realized, for example, for a set of parallel repulsive potential curves. These two points could explain why the relative energy positions of the primary molecular satellite states seem approximately to coincide with the corresponding atomic lines with respect to the dissociative double-ionization threshold. However, from the quality and resolution of our data this is by no means a proven statement, there is still the possibility that both groups of lines are basically unrelated, as one would expect at first. Figure 3 shows the discrete part of the low-energy electron spectrum together with known $\mathrm{O}^{*}$ and $\mathrm{C}^{*}$ energy levels [19-21] ordered along common configurations but without considering different coupling schemes. The correspondence of the strongest lines with assigned $\mathrm{O}^{*}$ energy levels is in most cases better than $0.05 \mathrm{eV}$, an agreement only found for two of the $C^{*}$ energy levels [19]. This is supported by the fact that evidence for most of the observed transitions has been seen recently in the dissociative single photoioniza-

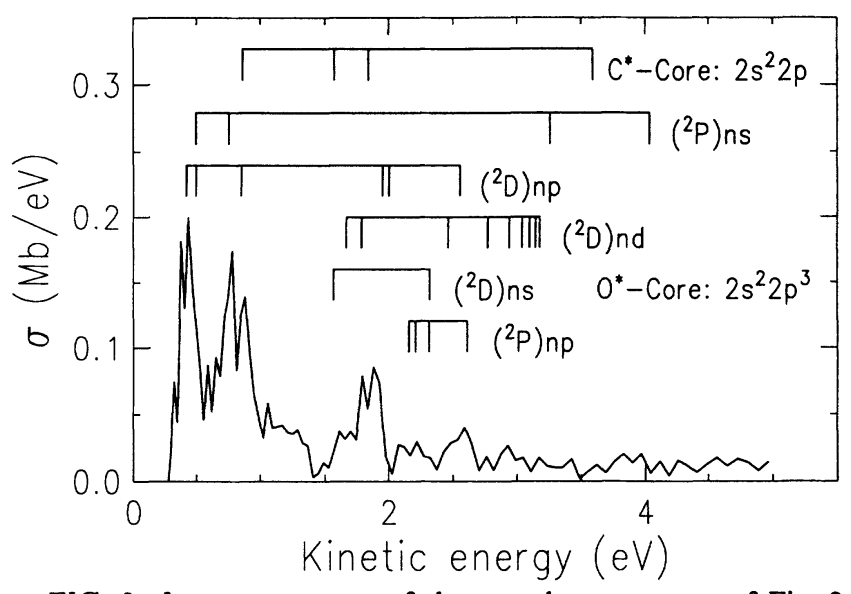

FIG. 3. Low-energy part of the complete spectrum of Fig. 2 together with depicted energy levels of $\mathrm{O}^{*}$ and $\mathrm{C}^{*}$ taken from Refs. [19-21]. The continuous background due to direct double ionization and eventual molecular Auger decay is subtracted.

tion of $\mathrm{O}_{2}[22,23]$. It is one of the surprising results of this study that valence two-hole-one-electron excitations in $\mathrm{CO}$ seem to be sufficiently localized to allow preferential fragmentation to $\mathrm{O}^{*}$ atoms. This process will also have substantial effect on the decay of core-electron excitations such as $\mathrm{C} 1 s \rightarrow \pi^{*}$ in CO [8] because the deexcitation of this resonance strongly populates the twohole-one-electron states investigated here [24,25]. The observed enhancement of the continuous part of the lowenergy spectrum is most likely due to molecular relaxation to the lowest $\mathrm{CO}^{2+}$ state before dissociation. Clearly, more detailed studies like electron-electron coincidence measurements are necessary to prove these assumptions.

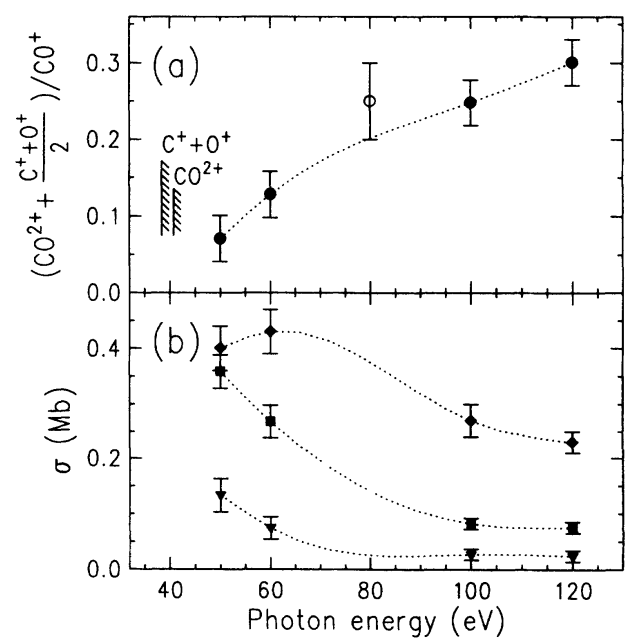

FIG. 4. (a) Branching ratio for double ionization including sequential dissociative double ionization relative to single ionization. (b) Partial cross section for the different double-ionization processes: direct double ionization (diamonds), indirect ionization via Auger decay (squares), and dissociative double ionization via atomic relaxation in the fragments (triangles). The open circle is from Ref. [5]. The dotted lines are drawn to guide the eye. 
Despite the unexpected strong appearance of two-step processes including discrete lines near threshold, direct double ionization still remains the dominant process contributing to the double ionization in the sudden limit. In contrast to the atomic case the continuous part of the complete valence spectrum is relatively undisturbed, facilitating the quantitative analysis of these direct doubleionization processes. In this analysis, the thresholds and relative intensities of these processes were taken in accordance with the kinetic-energy recoil distribution (KERD) data of Lablanquie et al. [5]. According to them, the lowest threshold for direct double ionization is the metastable $\mathrm{CO}^{2+}$ state at $40.75 \mathrm{eV}$. The kinetic-energy distribution of the direct two-electron emission processes was assumed to be in accord with the theoretical distribution curves derived by Chang and Poe [26], which fit the experimental data in helium fairly well [27]. A similar situation seems to be present in $\mathrm{CO}$, because the sum of the different distribution curves is, considering the limited photon resolution of our experiment, in surprisingly good agreement with the recorded data, as seen in Fig. 1. The quantitative analysis of our $\mathrm{CO}$ electron spectra gives partial cross sections for both direct and indirect processes shown in Fig. 4. The sum of the two processes for $\mathrm{CO}$ agrees reasonably with the ion-yield datum point of $\mathrm{La}-$ blanquie et al. [5] Theoretical data still do not exist for comparison.

Our results show that valence double ionization resulting from both direct and indirect processes, contributes up to $25 \%$ of all photoionization and accompanying dissocia- tion processes in the sudden limit. This is twice the value know for the isoelectronic neon [10], showing the increased role of electron correlations in the inner-valence regime of molecules.

In summary, we have observed direct evidence for sequential processes in dissociative valence double ionization yielding discrete electron lines at fixed, low kinetic energies. These lines are interpreted as atomic Auger lines predominantly from excited $\mathrm{O}^{*}$ states populated via fast dissociation of repulsive two-hole-one-electron satellite states. Contrary to expectations, decay to repulsive states of the undissociated doubly charged molecular ion is less important in this two-step process. Besides the strong occurrence of these sequential processes near threshold, the dominant process in the sudden limit is direct double ionization associated with continuous twoelectron emission. All double-ionization channels together make up to $25 \%$ of all ionization and dissociation events following photoionization of $\mathrm{CO}$ in the inner-valence region. Similar results have been obtained for molecular nitrogen and are expected also for other molecules.

We are indebted to Professor Sonntag and the members of his group for their assistance with the beam line and to Professor Cederbaum and Professor Schirmer for helpful discussions. This work was supported by the Bundesminister für Forschung und Technologie under Contract No. $05414 \mathrm{CAB} 7$ and by the Deutsche Forschungsgemeinschaft under Contract No. BE 860/2-4.
[1] L. S. Cederbaum, W. Domcke, and J. Schirmer, Adv. Chem. Phys. 65, 115 (1986).

[2] I. Nenner and J. A. Beswick, in Handbook of Synchrotron Radiation, edited by G. V. Marr (Elsevier, Amsterdam, 1987), Vol. 2.

[3] T. Masuoka and J. A. R. Samson, J. Chem. Phys. 74, 1093 (1981).

[4] T. Masuoka, J. Chem. Phys. 82, 3921 (1985).

[5] P. Lablanquie, J. Delwiche, M.-J. Hubin-Franskin, I. Nenner, P. Morin, K. Ito, J. H. D. Eland, J.-M. Robbe, G. Gandara, J. Fournier, and P. G. Fournier, Phys. Rev. A 40, 5673 (1989).

[6] P. Morin and I. Nenner, Phys. Rev. Lett. 56, 1913 (1986).

[7] P. Morin and I. Nenner, Phys. Scr. T17, 171 (1987).

[8] A. P. Hitchcock, P. Lablanquie, P. Morin, E. Lizon, A. Lugrin, M. Simon, P. Thiry, and I. Nenner, Phys. Rev. A 37, 2448 (1988).

[9] A. Svensson, E. A. Hughes, A. Baninchevich, S. D. Peyerimhoff, and B. A. Hess, J. Phys. B 24, 2997 (1991).

[10] U. Becker, R. Wehlitz, O. Hemmers, B. Langer, and A. Menzel, Phys. Rev. Lett. 63, 1054 (1989).

[11] S. D. Price and J. H. D. Eland, J. Phys. B 22, L153 (1989).

[12] A. A. Wills, A. A. Cafolla, A. Svensson, and J. Comer, J. Phys. B 23, 2013 (1990).

[13] J. Schirmer and O. Walter, Chem. Phys. 78, 201 (1983); J. Schirmer (private communication).

[14] U. Becker, D. Szostak, H. G. Kerkhoff, M. Kupsch, B. Langer, R. Wehlitz, A. Yagishita, and T. Hayaishi, Phys.
Rev. A 39, 3902 (1989).

[15] R. Bruhn, E. Schmidt, H. Schröder, B. Sonntag, A. Thevenon, G. Passereau, and J. Flamand, Nucl. Instrum. Methods 208, 771 (1983).

[16] W. Peatman, C. Carbone, W. Gudat, W. Heinen, P. Kuske, J. Pflüger, F. Schäfers, and T. Schroeter, Rev. Sci. Instrum. 60, 1445 (1989).

[17] S. Krummacher, V. Schmidt, F. Wuilleumier, J. M. Bizau, and D. Ederer, J. Phys. B 16, 1733 (1983).

[18] J. H. D. Eland, Photoelectron Spectroscopy (Butterworth, London, 1984).

[19] C. E. Moore, Atomic Energy Levels, U. S. National Bureau of Standards Special Publication No. 467 (U.S. GPO, Washington, D.C., 1949), Vol. 1.

[20] R. E. Huffman, J. C. Larrabee, and Y. Tanaka, J. Chem. Phys. 46, 2213 (1966).

[21] R. E. Huffman, J. C. Larrabee, and Y. Tanaka, J. Chem. Phys. 47, 4462 (1967).

[22] A. A. Cafolla, T. Reddish, and J. Comer, J. Phys. B 22, L273 (1989).

[23] A. A. Wills, A. A. Cafolla, and J. Comer, J. Phys. B (to be published).

[24] W. Eberhardt, E. W. Plummer, C. T. Chen, and W. K. Ford, Aust. J. Phys. 39, 853 (1986).

[25] W. Eberhardt, Phys. Scr. T17, 28 (1987).

[26] T. N. Chang and R. T. Poe, Phys. Rev. A 12, 1432 (1975).

[27] R. Wehlitz, F. Heiser, O. Hemmers, B. Langer, A. Menzel, and U. Becker, Phys. Rev. Lett. 67, 3764 (1991). 